Rev. Elev. Méd. vét. Pays trop., 1970, 23 (4) : 457-67

\title{
Etude, chez le zébu des zones tropicales, du pouvoir anthelminthique de deux composés dibenzeniques, dérivés bromés de la Salicylanilide
}

\author{
par M. GRABER et BIRGI (*)
}

\begin{abstract}
RESUME
L'auteur étudie le pouvoir anthelminthique de deux dérivés bromés de la Salicylanilide: I'Hilomid et son composant tribromé. Les deux médicaments ont un spectre d'activité étendu qui intéresse les Fasciola gigantica mûres âgées de plus de 77 semaines, les Paramphistomidés et les Gastrothylacidés adultes de la panse, Thysaniezia ovilla et des Nématodes tels que Bunostomum phlebotomum et Bosicola radiatum. Les médicaments sont administrés, sans mise à la diète préalable, à la dose de $50 \mathrm{mg} / \mathrm{kg}$ pour l'Hilomid et de $30-35 \mathrm{mg} / \mathrm{kg}$ pour le dérivé tribromé. Les doses ne sont pas répétées.

La marge de sécurité varie en fonction de l'âge et de l'état des animaux : elle paraît dans l'ensemble suffisante $(C / T=3$ à 5$)$.

Les résultats sont moins bons lorsqu'il s'agit de traiter des distomatoses récentes. Avec le Dérivé tribromé, les doses doivent être sensiblement augmentées, ce qui réduit d'autant le coefficient chimiothérapique. Dans ce cas. des précautions s'imposent( pesées des animaux et admin:stration d'une dose rigoureusement exacte).
\end{abstract}

\section{INTRODUCTION}

En Afrique centrale (Tchad, Nord-Cameroun et R.C.A.), les Trématodes gastriques et hépatobiliaires du zébu et du bœuf sont particulièrement fréquentes de la forêt équatoriale au Sahel, c'est-à-dire entre les isohyètes 650 et $1.700 \mathrm{~mm}$ (GRABER et collab., 1966; GRABER, 1969; BIRGI et GRABER, 1969; GRABER et collab., 1969).

De nombreux parasites, seuls ou en association, interviennent dont Dicrocoelium hospes, Fasciola gigantica, des Paramphistomidés $(P a-$ ramphistomum microbothrium, Cotylophoron cotylophorum, Calicophoron calicophorum, Calicophoron ijimai, Calicophoron raja, Ste-

(*) Institut d'Elevage et de Médecine vétérinaire des Pays tropicaux, Maisons-Alfort; Laboratoire de Farcha, Fort-Lamy, République du Tchad; Chaire de Parasitologie, Ecole nationale vétérinaire de Lyon. phanopharynx compactus, Bothriophoron bothriophoron) et des Gastrothylacidés (Carmyerius spatiosus, Carmyerius papillatus, Carmyerius parvipapillatus, Carmyerius gregarius et Carmyerius graberi). Les taux d'infestation sont élevés : 35 à 62 p. 100 pour Fasciola gigantica; 0,5 à 46 p. 100 pour Dicrocoelium hospes; 5 à 72 p. 100 pour les Paramphistomidés et 9 à 43 p. 100 pour les Gastrothylacidés.

Exerçant leur pouvoir pathogène sur des animaux sous-alimentés une bonne partie de l'année (en saison sèche), ces helminthes provoquent des pertes, directes et indirectes, sérieuses qu'il importe de limiter au maximum.

Actuellement, la prophylaxie tend, dans la nature, à détruire les vecteurs (Limnaea natalensis) et, chez l'hôte, les "Distomes " du foie et de la panse à l'aide d'anthelminthiques divers. En cette matière, depuis 10 ans, des progrès spectaculaires ont été réalisés en Eu- 
rope, en Amérique et en Australie. En Afrique, outre quelques essais effectués dans l'Est et au Sud du continent, des recherches ont été entreprises depuis 1964 au laboratoire de Farcha (République du Tchad) en vue de déterminer, chez le zébu, le pouvoir anthelminthique d'une dizaine d'antidistomiens récents. Deux d'entre eux qui sont connus depuis 1963 (LIENERT) ont retenu plus particulièrement l'attention : ce sont des composés dibenzéniques, dérivés bromés de là Salicylanilide.
Le premier est constitué par un mélange à parties égales de dibromo 4'5 Salicylanilide et de tribromo 3,4',5 Salicylanilide (= Hilomid $\left(^{(}\right.$) $=$ mitényl). Il a fait l'objet de nombreuses études, tant chez le mouton que chez le bœuf. Il se présente sous l'aspect d'une poudre blancgrisâtre, fine, inodore, renfermant 90 p. 100 de principes actifs. Le second est le dérivé tribromé seul, sous forme de comprimés de un gramme :

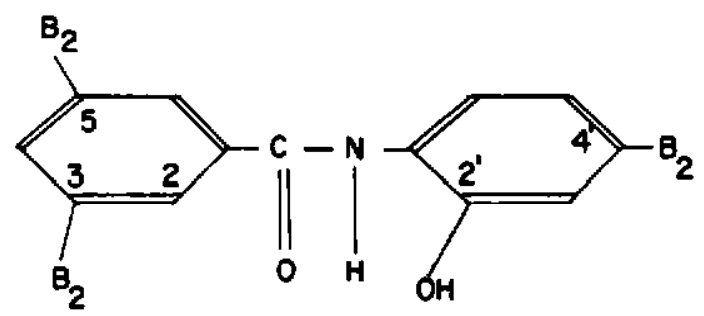

\section{MATERIEL ET METHODE}

\section{Les animaux}

86 animaux au total ont été mis en expérience en 1968-1969, dont 58 bouvillons et 28 vaches âgées. Les premiers, originaires de Fort-Lamy et de Bouar, pesaient en moyenne $107 \mathrm{~kg}$ (de 38 à 191) et les secondes $252 \mathrm{~kg}$ (de 165 à 379) $\left(^{2}\right)$.
Ils ont été choisis au hasard dans les troupeaux et l'échantillonnage est assez représentatif de ce que l'on peut trouver en zone sahélienne.

Leur état d'entretien était, dans l'ensemble, très moyen, sinon mauvais.

Plusieurs lots ont été constitués.

TABLEAU $\mathrm{N}^{\circ} \mathrm{I}$

\begin{tabular}{|l|c|c|c|c|}
\hline & \multicolumn{2}{|c|}{ Bouvilions } & \multicolumn{2}{c|}{ Vaches âgées } \\
\cline { 2 - 5 } & Dértvé tribromé & Hilomid & Hilomid & Dérivé tribromé \\
\hline $\begin{array}{l}\text { Essals thérapeutiques } \\
\text { proprement dits : }\end{array}$ & 8 & 3 & 8 & 12 \\
- Térnoirs & 2 & 7 & 1 & 3 \\
$\begin{array}{l}\text { Essais sur F.gígantíca } \\
\text { imnatures }\end{array}$ & 9 & 1 & - & - \\
- Témoins & 6 & 1 & - & 3 \\
\hline
\end{tabular}

(1) Astra Pharmaceuticals, Suède; Sovétal, France.

(2) Bœufs Kouris et métis zébus-Kouris (Massakory). 


\section{Les parasites}

Les helminthes suivants ont été recueillis à l'autopsie :

TABLEAU $N^{\circ} I I$

Farasites en cause

\begin{tabular}{|c|c|c|c|c|c|}
\hline \multirow{2}{*}{ Espèces parasites } & \multicolumn{2}{|c|}{ Vaches âgées } & \multicolumn{2}{|c|}{ Bouvillons } & \multirow{2}{*}{ Total } \\
\hline & Traitēes & Témoing & Traitês & Témoins & \\
\hline Dicroccelium hospes & - & - & 4 & 4 & 8 \\
\hline Easciola gigantica & 17 & 2 & $\cdot 13$ & 10 & 42 \\
\hline Cotylopinoron cotyzopkorw. & - & - & 1 & 3 & 4 \\
\hline Pcranphistomon microbothrium & 4 & 2 & - & 2 & 8 \\
\hline Paramphistomum sp. & $B$ & - & 2 & - & 10 \\
\hline Cormyerius spatiosus & 1 & - & - & - & 1 \\
\hline Camperius papiziatus & 2 & 1 & - & - & 3 \\
\hline Camyerius parvi papilzatus & 1 & - & - & - & 1 \\
\hline Carmyerius graberi & - & 1 & - & - & 1 \\
\hline Schistoroma bovis & 21 & 3 & 7 & 2 & 33 \\
\hline Moniezia benederi & 2 & - & 1 & 1 & 4 \\
\hline Cysticercus bovis & - & - & 4 & - & 4 \\
\hline Echinococcus polymorphus & 1 & - & - & - & 1 \\
\hline Bosioola radiatum & 5 & - & 13 & 4 & 23 \\
\hline Burostcmum phlebotomum & - & - & 10 & 4 & 14 \\
\hline Cooperia punctata & 6 & 2 & 11 & 2 & 21 \\
\hline Cooperia pectinata & 6 & 1 & 15 & 2 & 24 \\
\hline Haemonous contcrtus & - & - & 7 & 4 & 11 \\
\hline Setaria labiato papilizosa & 14 & 3 & 8 & 3 & 28 \\
\hline onchocerca-gutturosa & 19 & 1 & 4 & - & 24 \\
\hline Cnchocerca cmiliata & 11 & - & 2 & - & 13 \\
\hline Euakleyuris globulcsa & - & - & 2 & - & 22 \\
\hline
\end{tabular}

\section{La technique}

Pour les Cestodes, les Nématodes et les Trématodes adultes, elle a été décrite à de nombreuses reprises (I.E.M.V.T., 1969). Nous n'y reviendrons donc pas.

En ce qui concerne Fasciola gigantica immatures, quelques points demandent à être précisés. Des bouvillons d'une centaine de kilogrammes et indemnes de distomatose reçoivent de 100 à 4.000 métacercaires, âgées de 7 à 13 jours, provenant de Limnées d'élevage ou de mollusques parasités récoltés sur le terrain (lac de Fianga).

Les animaux sont traités 41-44, 58-61, 61-68 et 77-94 jours après l'infestation expérimentale. Les dérivés bromés de la Salicylanilide ayant tendance à s'éliminer très vite, les autopsies sont effectuées 3 à 4 jours après l'administration du médicament. Les foies sont retirés dès la mort de l'animal et les jeunes douves extraites immédiatement, en commençant par la capsule de Glisson et le parenchyme hépatique et en terminant par les canaux biliaires. Les parasites sont alors plongés dans de l'eau tiède ou dans de la bile de bœuf : leurs mouvements sont, la plupart du temps, bien visibles sous la loupe. Puis, les Distomes sont fixés au formol: s'ils sont encore vivants, ils prennent un aspect plissé caractéristique. Dans le cas contraire, ils sont en extension complète.

Parfois (faibles infestations de 6-7 semaines), le parasite se comporte comme un corps étranger. Le tissu hépatique réagit rapidement et 
tend à lyser le parasite : celui-ci, qui est normalement blanc laiteux, semi-transparent, s'opacifie et devient blanc grisâtre. Il est replié sur lui-même.

Chaque bouvillon ou chaque lot est accompagné d'un témoin ayant absorbé le même nombre de métacercaires.

Le protocole expérimental s'apparente à celui décrit par BORAY (1963 et 1967) qui, chez le mouton, consiste à infester plusieurs lots, à les traiter $28,42,56$ et 84 jours plus tard, en y adjoignant un certain nombre de témoins. L'autopsie est faite deux semaines après le dernier traitement, c'est-à-dire 98 jours après l'infestation initiale. La comparaison entre la moyenne du nombre de douves immatures chez les ovins traités et chez les témoins donne le pourcentage d'efficacité.

Malheureusement, chez les bovins, cette méthode semble difficile à mettre en œuvre: dans cette espèce, le nombre de douves recueillies à l'autopsie étant sujet à d'amples fluctuations, on risque de comparer deux séries de chiffres (traités et témoins) qui, au départ, sont très dissemblables, donc de fausser les résultats dans un sens ou dans l'autre. Aussi, la technique de Boray a-t-elle été adaptée.

Les anthelminthiques ont été administrés dans de l'eau «à la bouteille", sans rrise à la diète préalable.

Les doses n'ont pas été répétées.

\section{RESULTATS}

\section{Action sur les Trématodes}

1-1. Dicrocoelium hospes (canaux biliaires).

L'Hilomid est dépourvu de tout pouvoir anthelminthique aux doses de 40 et de $60 \mathrm{mg} /$ $\mathrm{kg}$ : la moyenne du nombre de Dicrocoelium (40 et 20 ) est toujours supérieure à ce qu'elle est chez les témoins $(5,5)$.

1-2. Schistosoma bovis (veines mésentériques et hépatiques).

Les deux médicaments sont totalement inefficaces, même à des doses très élevées de l'ordre de $225 \mathrm{mg} / \mathrm{kg}$ (Hilomid) et de $100 \mathrm{mg} / \mathrm{kg}$ (dérivé tribomé).
1-3. Fasciola gigantica adultes (voies biliaires) et immatures (parenchyme hépatique).

$$
\begin{aligned}
& \text { tableau } n^{\circ} \quad 3: \text { adultes } \\
& \text { tableau } n^{0} \quad 4: \text { immatures. }
\end{aligned}
$$

Chez le zébu, le dérivé tribromé détruit Fasciola gigantica adulte et mûre à $20 \mathrm{mg} / \mathrm{kg}$. Avec l'Hilomid, il faut au moins $40 \mathrm{mg} / \mathrm{kg}$, dose nettement plus élevée que celles recommandées par LIENERT (1963 - $30 \mathrm{mg} / \mathrm{kg}\left({ }^{3}\right)$, ONO et collab. $(1964-25 \mathrm{mg} / \mathrm{kg})$ et NAKAMURA et collab. (1966 - 25 mg/ kg), dans les cas de distomatose bovine à Fasciola hepatica. En outre, avec Fasciola gigantica, il ne semble pas nécessaire de renouveler la dose de 30 (CHIROL et DAVID, 1967) ou de $40 \mathrm{mg} / \mathrm{kg}$ (EUZEBY, 1968; DANNONAY, 1968) à 10 ou 24 heures d'intervalïe.

Il est bon de rappeler que, chez des moutons hébergeant Fasciola hepatica ou Fasciola gigantica, le médicament est administré en une seule prise à la dose de 30 (BORAY et collab., 1965 et 1966; Anonyme 1966; HILDEBRANDT, 1968) ou de $40 \mathrm{mg} / \mathrm{kg}$ (DANNONAY, 1968).

Par contre, lorsque les Distomes sont immatures, c'est-à-dire au cours de la phase prépatente de la maladie, les composés bromés de la Salicylanilide semblent beaucoup moins actifs. On ne possède actuellement que peu de renseignements, sauf chez le mouton (BORAY et collab., 1965; BORAY et collab., 1967; HILDEBRANDT, 1968; DANNONAY, 1968). Les essais réalisés à Farcha, chez le zébu, montrent que le dérivé tribromé à $20 \mathrm{mg} / \mathrm{kg}$ est capable de tuer la totalité des douves âgées de 77 jours et plus. Entre 58 et 68 jours, l'efficacité n'est plus que de $26 \mathrm{p}$. 100 à $25 \mathrm{mg} / \mathrm{kg}$ et de 53 p. 100 à $30 \mathrm{mg} / \mathrm{kg}$. A six semaines, le pourcentage de douves mortes est d'environ $71 \mathrm{p} .100$ à $40-45 \mathrm{mg} / \mathrm{kg}$.

Pour les Fasciola de moins de 11 semaines, il faut donc augmenter considérablement la dose thérapeutique normale (au moins $\times 1,5$ ), ce qui est le cas également d'autres antidistomiens récents (Nitroxynil - Bilevon $\mathrm{M}$ ).

De son côté, l'Hilomid à $35 \mathrm{mg} / \mathrm{kg}$ est actif sur les Fasciola gigantica de 76-83 jours.

(3) Diaphène: 3 parties du composé trıbromé et une partie du dérivé dibromé. 


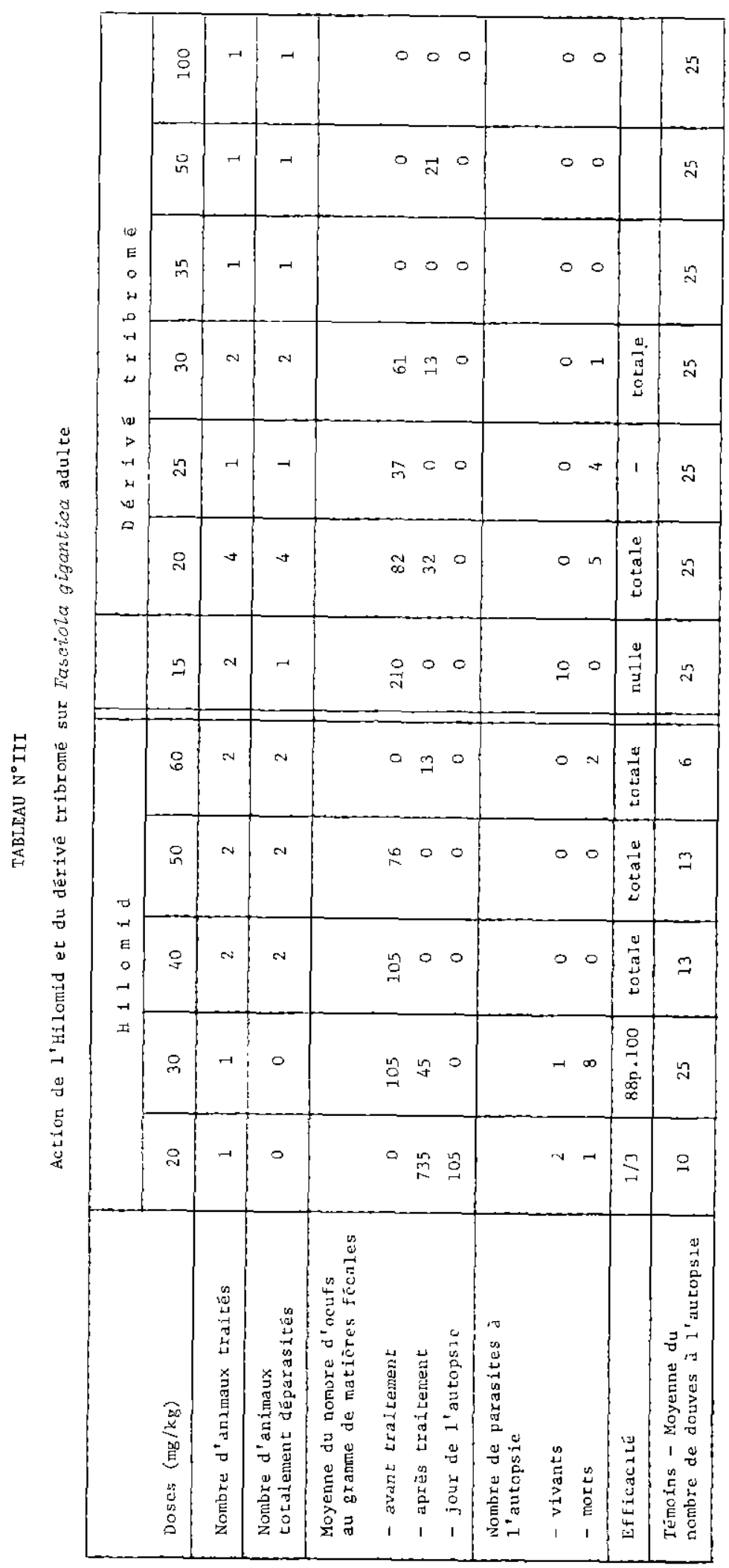


TABLEAU $\mathbb{N}^{\circ}$ IV

Action de 1'Hilomid et du dértvê tribromé sur Fasciola gigantica immature.

\begin{tabular}{|c|c|c|c|c|c|c|c|c|}
\hline \multirow[b]{2}{*}{$\begin{array}{l}\text { Bouvillon } \\
\qquad \mathrm{N}^{\circ}\end{array}$} & \multirow{2}{*}{\begin{tabular}{l}
\multicolumn{1}{c}{ Nombre de } \\
mêtacercaires \\
administrées
\end{tabular}} & \multirow{2}{*}{$\begin{array}{l}\text { Ages des } \\
\text { douves } \\
\text { (en jours) }\end{array}$} & \multicolumn{4}{|c|}{ Bouvillons traités } & \multicolumn{2}{|c|}{ Bouvillons témoins } \\
\hline & & & $\begin{array}{l}\text { Doses } \\
\mathrm{mg} / \mathrm{kg}\end{array}$ & $\begin{array}{l}\text { Nombre de } \\
\text { douves à } \\
1 \text { 'autopsie }\end{array}$ & $\begin{array}{l}\text { Taille des } \\
\text { parasites } \\
\text { (en num) }\end{array}$ & $\begin{array}{c}\text { Efficacitë } \\
\text { (p.100) }\end{array}$ & $\begin{array}{l}\text { Nombre de } \\
\text { douves a } \\
1 \text { 'autopsie }\end{array}$ & $\begin{array}{l}\text { Taille des } \\
\text { douves } \\
\text { (en mm) }\end{array}$ \\
\hline \multicolumn{2}{|c|}{ Dérivê tribromé } & & & & & & & \\
\hline 39 & $200(1)$ & 41 & 40 & $\begin{array}{l}5 \text { mortes } \\
\text { )1 vivante }\end{array}$ & $5,5-7 \times 1$ & $\begin{array}{l}\text { 41-44 jours } \\
\text { en moyenne }\end{array}$ & - & - \\
\hline 44 & $100(1)$ & 44 & 45 & $\left\{\begin{array}{l}7 \text { mortes } \\
44 \text { vivantes }\end{array}\right.$ & $\begin{array}{r}8,5-10 \times \\
1-2\end{array}$ & 70,6 p.100 & - & - \\
\hline 41 & $100(1)$ & 44 & - & - & - & - & 10 & $8,5-10,5 \times 1-2$ \\
\hline 36 & 500 & 61 & 30 & $\begin{array}{l}130 \text { vivantes } \\
\text { } 46 \text { mortes }\end{array}$ & $\begin{array}{c}14-22 \times \\
2,1-4\end{array}$ & $\begin{array}{l}58-61 \text { jours } \\
\text { en moyerne }\end{array}$ & - & \\
\hline 30 & 500 & 58 & 50 & $\left\{\begin{array}{l}36 \text { vivantes } \\
144 \text { mortes }\end{array}\right.$ & $\begin{array}{c}7,5-12 \times \\
1-2,5\end{array}$ & $53,3 \mathrm{p} .100$ & - & - \\
\hline 29 & 500 & 56 & - & - & - & - & 96 & $8-12 \times 1,2-2,5$ \\
\hline 33 & 500 & 68 & 25 & $\left\{\begin{array}{l}42 \text { vivantes } \\
5 \text { mortes }\end{array}\right.$ & $\begin{array}{c}10,5-16 \times \\
2-3,2\end{array}$ & $\begin{array}{l}61-68 \text { jours } \\
\text { en moyenne }\end{array}$ & - & - \\
\hline 35 & 500 & 61 & 25 & $\left\{\begin{array}{l}121 \text { vivantes } \\
52 \text { mortes }\end{array}\right.$ & $\begin{array}{l}8-20 \times 1,5 \\
-2,5\end{array}$ & 25,9 p. 100 & - & - \\
\hline 32 & 500 & 68 & - & - & - & - & 213 & $13-22 \times 2,5$ \\
\hline 25 & 500 & 57 & 20 & 165 vivantes & $10-16 \times 1,5-2,5$ & nulle & - & - \\
\hline 38 & 500 & 51 & - & - & - & - & 52 & $6,5-10 \times 0,9-1,8$ \\
\hline 7 & $1500(2)$ & $80-94$ & 20 & 216 mortes & $27-35 \times 3,5-4$ & totale & - & - \\
\hline 4 & $1500(2)$ & $72-87$ & - & - & - & - & 423 & $12-30 \times 2,-4,5$ \\
\hline 10 & 3000 (2) & $77-92$ & 20 & 586 mortes & $26-31 \times 3-3,5$ & totale & - & - \\
\hline 11 & 3000 (2) & $74-86$ & - & - & - & - & 900 & $13-25 \times 2-4$ \\
\hline Hilomid & & & & & & & & \\
\hline 20 & 4000 (2) & $76-83$ & 35 & 948 mortes & $22-23 \times 3$ & totale & - & - \\
\hline 13 & $4000(2)$ & $78-91$ & - & - & - & - & 1654 & $28-31 \times 3,5-5$ \\
\hline
\end{tabular}

(1) Mêtacercatres provenant de Limnées recuelilies sur les bords du lac de Fianga:

(2) en 2 fois à $13-15$ jours d'intervalle. 
1-4. Paramphistomidés et Gastrothylacidés adultes de la panse.

ONO et collaborateurs (1964), sur examens coproscopiques, avaient déjà remarqué que le nombre d'œufs de Paramphistomidés avait tendance à diminuer après traitement à l'Hilomid (25 $\mathrm{mg} / \mathrm{kg}$ ).
Les résultats qui figurent au tableau $n^{\circ} 5$ confirment cette observation.

La quasi-totalité des Paramphistomidés et des Gastrothylacidés mûrs localisés dans la panse disparaissent à $50 \mathrm{mg} / \mathrm{kg}$, dans le cas de l'Hilomid, et vers $30-35 \mathrm{mg} / \mathrm{kg}$, dans le cas du dérivé tribromé.

Action de l'Hilonid et du dêr lvé tribromé sur divers Paramphistomidés et Gastrothylacldés de la panse.

\begin{tabular}{|c|c|c|c|c|c|c|c|c|c|}
\hline \multirow[b]{2}{*}{ Doses (mg $/ \mathrm{kg}$ ) } & \multicolumn{4}{|c|}{$\mathrm{H} I \mathrm{i}$ a m $\mathrm{i} d$} & \multicolumn{5}{|c|}{ Dérivé tribromé } \\
\hline & 30 & 40 & 50 & 60 & 20 & 25 & 30 & 35 & 80 \\
\hline Nombre d'animaux traités & 1 & 3 & 3 & 1 & 2 & 1 & 5 & I & 1 \\
\hline $\begin{array}{l}\text { Nombre d'antmaux } \\
\text { totalement déparasitês }\end{array}$ & 0 & 1 & 3 & 1 & 0 & 0 & 4 & 1 & 1 \\
\hline $\begin{array}{l}\text { Moyenne du nombre d'oeufs } \\
\text { au gramme de matiêres fécales } \\
\text { - avant traltement } \\
\text { - après traltement } \\
\text { - jour de } 1^{\prime} \text { autopsie }\end{array}$ & $\begin{array}{r}0 \\
131 \\
0\end{array}$ & $\begin{array}{r}210 \\
105 \\
0\end{array}$ & $\begin{array}{r}542 \\
183 \\
0\end{array}$ & $\begin{array}{r}1235 \\
120 \\
0\end{array}$ & $\begin{array}{r}105 \\
26 \\
105\end{array}$ & $\begin{array}{r}105 \\
0 \\
0\end{array}$ & $\begin{array}{r}140 \\
44 \\
0\end{array}$ & $\begin{array}{r}70 \\
105 \\
0\end{array}$ & $\begin{array}{r}0 \\
140 \\
0\end{array}$ \\
\hline $\begin{array}{l}\text { Parasites vivants à } \\
\text { l'autopste }^{\prime} \\
\text { (Poids moyen en g.) }\end{array}$ & $\begin{array}{l}0,5 \\
(1)\end{array}$ & $\begin{array}{c}3 \\
(2)\end{array}$ & 0 & 0 & $\begin{array}{c}6 \\
(4)\end{array}$ & $\begin{array}{l}0,01 \\
(7)\end{array}$ & $\begin{array}{l}0,01 \\
(3)\end{array}$ & 0 & 0 \\
\hline Efficacitë & nulle & $1 / 3$ & totale & - & nu11e & faible & $4 / 5$ & totale & - \\
\hline $\begin{array}{l}\text { Témolns - Poids moyen } \\
\text { (en grammes) }\end{array}$ & $\begin{array}{l}1,2 \\
(6)\end{array}$ & $\begin{array}{l}31 \\
(6)\end{array}$ & $\begin{array}{r}31 \\
(6)\end{array}$ & $\begin{array}{l}1,2 \\
(6)\end{array}$ & $\begin{array}{l}106 \\
(5)\end{array}$ & $\begin{array}{l}106 \\
(5)\end{array}$ & $\begin{array}{l}106 \\
(5)\end{array}$ & $\begin{array}{c}106 \\
(5)\end{array}$ & $\begin{array}{l}106 \\
(5)\end{array}$ \\
\hline
\end{tabular}

(1) Poramphistomum microbothrium 1mmatures;

(2) Carmyerius papiziatus mûrs et Cotylophoron cotyzophomum immatures;

(3) Carmyerius popizzatus imnatures:

(4) Paranphistomum microbothrium mûrs;

(5) Paramphistomw microbothrium et Camyerius graberi mûrs;

(6) Paramphistomum microbothrium et Cotulopinoron cotylophorum mûrs;

(7) Carmmerius spatiosus mûrs.

\section{Action sur les Cestodes}

\section{2-1. Intestin}

Le dérivé tribromé chasse Thysaniezia ovilla vers $20-25 \mathrm{mg} / \mathrm{kg}$ et l'Hilomid vers $60 \mathrm{mg} / \mathrm{kg}$. Le nombre d'animaux mis en expérience est cependant trop réduit pour que des conclusions définitives puissent être tirées.

\section{2-2. Muscle}

Cysticercus bovis résiste à tous les traitements (dérivé tribromé).

\section{Action sur les Nématodes}

3-1. Les deux anthelminthiques sont sans effet sur Cooperia punctata, Cooperia pectinata et Buckleyuris globulosa de l'intestin, ainsi que sur les Filaires du péritoine, de l'aorte et du ligament cervical.

\section{3-2. Haemoncus contortus (caillette)}

Les résultats sont irréguliers. L'Hilomid à $60 \mathrm{mg} / \mathrm{kg}$ et le dérivé tribromé, à $25-30 \mathrm{mg} / \mathrm{kg}$, assurent l'élimination d'un certain nombre d'Haemoncus (de 0 à 80 p. 100). 
3-3. Bunostomum phlebotomum (duodénum) et Bosicola radiatum (gros intestin)

Ils sont tous deux sensibles à l'action de l'Hilomid et du dérivé tribromé, le premier nettement plus que le second. Les formes $\mathrm{L}_{\breve{a}}$ de Bosicoia radiatum, encore immatures (tableau $\mathrm{n}^{\circ} 7$ ) paraissent un peu plus résistantes.
Les doses recommandées sont de $40-50 \mathrm{mg} /$ $\mathrm{kg}$ (Hilomid) et de $25-30 \mathrm{mg} / \mathrm{kg}$ (dérivé tribromé).

Les larves $\mathrm{L}_{4}$ sous muqueuses $\left({ }^{4}\right)$ ne sont pas touchées.

(4) Bosicola radiatum.

TABLEAU $\mathrm{N}^{\circ} \mathrm{VI}$

Action de l'Hilomld et du dérivê tribromé sur Bunostonum phlebotorman adulte.

\begin{tabular}{|c|c|c|c|c|c|}
\hline \multirow[b]{2}{*}{ Doses (mg/kg) } & \multicolumn{3}{|c|}{ II $\mathrm{i} 10 \mathrm{~m} \mathrm{id}$} & \multicolumn{2}{|c|}{ Dërivé tribromé } \\
\hline & 50 & 60 & 80 & 20 & 30 \\
\hline Nombre d'an1maux traités & 3 & 1 & 1 & 3 & 2 \\
\hline $\begin{array}{l}\text { Nombre d'animaux } \\
\text { totalement dẹparasités }\end{array}$ & 2 & 1 & 1 & 3 & 2 \\
\hline $\begin{array}{l}\text { Nombre total de parasites } \\
\text { êliminês }\end{array}$ & 5 & 6 & 1 & 13 & 8 \\
\hline $\begin{array}{l}\text { Nombre total de parasites } \\
\text { restant à } 1^{\prime} \text { autopsie }\end{array}$ & 1 & 0 & 0 & 0 & 0 \\
\hline Efficacité & $84 \mathrm{p} .100$ & - & - & totale & totale \\
\hline $\begin{array}{l}\text { Têmoins - Moyenne du } \\
\text { nombre de parasites }\end{array}$ & 15 & 15 & 15 & 4 & 4 \\
\hline
\end{tabular}

TABLEAU $\mathrm{N}^{\circ} \mathrm{VII}$

Action de l'Hilomid et du dérivé trubromé sur Bosiaota radiatum.

\begin{tabular}{|c|c|c|c|c|c|c|c|c|c|}
\hline \multirow[b]{2}{*}{ Doses (mg $/ \mathrm{kg})$} & \multicolumn{5}{|c|}{$\mathrm{H} i \mathrm{l} \circ \mathrm{m} \mathrm{id}$} & \multicolumn{4}{|c|}{ Dërivé tribromé } \\
\hline & 20 & 40 & 50 & 60 & 80 & 20 & 25 & 30 & 35 \\
\hline Nombre d'animaux traitês & 1 & 2 & 3 & 1 & 1 & 3 & 1 & 5 & 1 \\
\hline $\begin{array}{l}\text { Nombre d'animaux } \\
\text { totalement dêparasitếs }\end{array}$ & 1 & 2 & 3 & 1 & 1 & 2 & 0 & 4 & 1 \\
\hline $\begin{array}{l}\text { Moyeme du nombre de } \\
\text { larves L3 (3) }\end{array}$ & & & & & & & & & \\
\hline - avant traitement & - & - & 1 & - & - & 1 & - & 3 & - \\
\hline - après traitement & - & - & 0 & - & - & 0 & - & 7 & - \\
\hline - jour de 1 'autopsie & - & - & 0 & - & - & 0 & - & 0 & - \\
\hline $\begin{array}{l}\text { Nombre total de } \\
\text { parasites êliminês }\end{array}$ & 1 & 76 & 5 & 18 & 130 & 8 & 0 & $\begin{array}{r}72 \\
(2) \\
\end{array}$ & 1 \\
\hline $\begin{array}{l}\text { Nombre total de parasites } \\
\text { restant à l'autopsie }\end{array}$ & 0 & 0 & 0 & 0 & D & $\begin{array}{r}20 \\
(1) \\
\end{array}$ & 1 & 1 & 0 \\
\hline Efficacité & - & totale & totale & totale & totale & $\begin{array}{c}25,8 \mathrm{p} \\
100\end{array}$ & - & $\begin{array}{c}93,1 \mathrm{p} \cdot \\
100\end{array}$ & - \\
\hline $\begin{array}{l}\text { Témoins - Moyerne du } \\
\text { nombre de parasites }\end{array}$ & 20 & 38 & 13 & 20 & 20 & 59 & 2 & 59 & 59 \\
\hline
\end{tabular}

(1) Adultes 1matures;

(2) dont 3 adul tes immatures;

(3) dans une goutte de suspension provenant des boittes de Pétri. 
4. En définitive, l'Hilomid et le dérivé tribromé font preuve d'une polyvalence assez remarquable puisque en une seule prise, ils sont susceptibles d'atteindre Fasciola gigantica des voies biliaires, les Paramphistomidés et les Gastrothylacidés adultes de la panse, certains Cestodes de l'intestin, des Nématodes du duodénum ( $B$. phlebotomum) et du gros intestin (Bosicola radiatum). Haemoncus contortus de la caillette n'est que partiellement et irrégulièrement détruit.

Les doses les meilleures sont environ de $50 \mathrm{mg} / \mathrm{kg}$ pour l'Hilomid et de $30-35 \mathrm{mg} / \mathrm{kg}$ pour le dérivé tribromé. Cependant, en cas de distomatose récente, elles devront être sérieusement augmentées (le nombre de Fasciola de 6 semaines tuées par le dérivé tribromé n'est que de 71 p. 100 à la dose de $40-45 \mathrm{mg} / \mathrm{kg}$ ).

\section{MODE D'ACTION}

L'Hilomid est absorbé au niveau de l'intestin, puis il passe dans la circulation et de là, dans le foie, le rein et le tissu musculaire. Il s'élimine par l'urine, le foie et les matières fécales.

L'efficacité de l'anthelminthique est fonction de sa concentration dans ia bile. Chez le mouton, elle est encore importante 20 heures après l'administration du médicament. Chez le bovin, par contre, elle diminue rapidement et, au bout de 16 heures, elle n'est pius que de $8-10 \mathrm{ppm}$, ce qui explique pourquoi, dans cette espèce, on recommande de renouveler le traitement à 12-24 heures d'intervalle (CHIROL et DAVID, 1967; EUZEBY, 1968).

In vitro, Fasciola hepatica est tuée dans un délai compris entre 30 minutes et 3 heures.

In vivo, chez le zébu, les douves adultes sont attaquées très rapidement. Au bout de 24-36 heures, elles deviennent diaphanes et de larges plages transparentes apparaissent latéralement ou vers l'extrémité postérieure. Peu à peu, les Distomes prennent une couleur jaune d'or ou verdâtre. Dans 85 p. 100 des cas, l'expulsion des Trématodes est achevée cinq jours après le traitement.

Lorsqu'il s'agit de Paramphistomidés et de Gastrothylacidés, la coproscopie se négative en 3 ou 4 jours.
Quant à l'élimination des Anoplocéphalidés, des Bunostomes, des Esophagostomes et des Haemoncus, elle demande, la plupart du temps, 24 à 48 heures.

D'une façon générale, les dérivés bromés de la Salicylanilide permettent de chasser les Helminthes en un laps de temps relativement court (5 jours, contre 7-9 jours avec le Zanil ou le Bilevon R.) ${ }^{5}$ ).

\section{TOXICITE}

Des doses progressivement croissantes ont été administrées à des bouvillons et à de vieux animaux.

L'Hilomid est, en général, bien toléré jusqu'à $150 \mathrm{mg} / \mathrm{kg}$. Tout au plus, note-t-on un certain ramollissement des selles particulièrement accusé chez les jeunes de moins de $80 \mathrm{~kg}$.

Les premiers accidents mortels se produisent à partir de $150 \mathrm{mg} / \mathrm{kg}$ chez les bouvillons dont l'état est le plus mauvais. On observe de violentes coliques accompagnées d'une très forte dyspnée, de ptyalisme et de trémulations musculaires. L'appétit disparaît. Une diarrhée profuse, nauséabonde suit presque immédiatement l'administration du médicament. La mort survient en moins de 4 jours. Chez les survivants, les symptômes s'estompent progressivement. Tout est redevenu normal une semaine plus tard.

Le Dérivé tribromé semble mieux supporté par les bouvillons que par les vieilles vaches (tableau $\mathrm{n}^{\circ} 8$ ). Dans le premier cas, on peut aller jusqu'à $175 \mathrm{mg} / \mathrm{kg}$ sans inconvénients majeurs. Les coliques et la diarrhée ne durent que 48 heures. L'amaigrissement est modéré ( $-1,5$ p. 100 en trois semaines). Dans le second cas, vers $100 \mathrm{mg} / \mathrm{kg}$, une paralysie générale šinstalle 48 heures après le traitement: elle entraîne l'abattage des animaux.

Les lésions n'ont rien de caractéristique : ce sont celles de néphrite aiguë hémorragique, de congestion pulmonaire et de congestion intestinale avec, souvent, dimportantes hémorragies.

(a) Dérivés chlorés et nitrés de la Salicylanilide 
TABLEAU $\mathbb{N}^{\circ}$ VIII

Toxicité de l'Hilomid et đu dérivé tribromé

\begin{tabular}{|c|c|c|c|c|}
\hline \multirow{2}{*}{$\begin{array}{l}\text { Doses } \\
\text { (mg/kg) }\end{array}$} & \multicolumn{2}{|c|}{$\mathrm{H} i \mathrm{l} O \mathrm{mid}$} & \multicolumn{2}{|c|}{ Dérive tribromë } \\
\hline & Bouvillons & Vaches âgëes & Bouvilions & Vaches âgées \\
\hline de 15 à 50 & $\begin{array}{l}\text { à } 50 \mathrm{mg} / \mathrm{kg} \text {, selles } \\
\text { ramollies chez les } \\
\text { très jeunes animaux }\end{array}$ & blen supporté & bten supportê & bien supportê \\
\hline de 50 à 80 & $" \quad "$ & $"$ & $"$ & $"$ \\
\hline 100 & " $\quad "$ & " & coliques-diarrhêe & colíques-dtarrhée \\
\hline 125 & $" \quad "$ & $"$ & $"$ & mort en 28 heures \\
\hline 150 & 1 thort sur 2 & $"$ & $"$ & $"$ \\
\hline 175 & $" 1$ & $"$ & $"$ & $"$ \\
\hline 200 & $\begin{array}{l}1 \text { mort sur } 2 \\
\text { Anorexie-diarrhëe }\end{array}$ & $\begin{array}{c}1 \text { mort en } 48 \\
\text { beures }\end{array}$ & $"$ & $"$ \\
\hline 225 & $\begin{array}{l}\text { Amaigrissement et } \\
\text { diarrhée }\end{array}$ & & & \\
\hline
\end{tabular}

\section{CONCLUSIONS}

Une expérience réalisée dans la région de Fort-Lamy et comportant l'autopsie de 86 zébus d'âge et de poids différents a permis de comparer le pouvoir anthelminthique de deux dérivés bromés de la Salicylanilide : l'Hilomid et son composant tribromé.

Les deux médicaments font preuve d'une certaine polyvalence, puisqu'à des doses de $50 \mathrm{mg} / \mathrm{kg}$ (Hilomid) et de $30-35 \mathrm{mg} / \mathrm{kg}$ (dérivé tribromé), ils sont capables de détruire dans leur quasi-totalité les associations à base de Trématodes (Fasciola gigantica de plus de 11 semaines; les Paramphistomidés et les Gastrothylacidés adultes de la panse), de Cestodes (Thysaniezia ovilla) et de Nématodes (Bunostomum phlebotomum et Bosicola radiatum). Dicrocoelium hospes, Schistosoma bovis, Cooperia punctata, Cooperia pectinata et diverses filaires résistent à tous les traitements. L'action sur Haemoncus contortus est irrégulière.
Les douves immatures sont beaucoup plus résistantes et, à six semaines, le dérivé tribromé n'en tue que 70 p. $100(40-45 \mathrm{mg} / \mathrm{kg})$.

Les anthelminthiques sont administrés sans mise à la diète préalable et les doses ne sont pas répétées.

L'élimination des parasites est, dans la majorité des cas, terminée en cinq jours.

La toxicité varie en fonction de l'âge et de l'état général des zébus traités. Les vieux animaux sont, dans l'ensemble, plus sensibles que les jeunes. L'index thérapeutique est voisin de 3 (Hilomid) et de 3-5 (dérivé tribromé), ce qui laisse, en principe, une marge de sécurité suffisante. Cependant, le traitement des distomatoses récentes exigeant des quantités de médicaments bien supérieures, la dose thérapeutique se rapproche alors de la dose toxique. Des précautions sont à prendre (pesée des animaux, notamment).

\section{SUMMARY}

Study in tropical zebu cattle of the anthelmintic power of two dibenzenic compounds, Salicylanilide bromine derived

The author points out the anthelmintic power of two Salicylanilide bromine derived: Hilomid and its tribromine compound.

These drugs have a large spectrum of activity covering full-grown Fasciola gigantica (more than 77 days), Paramphistomidae and Gastrothylacidae of the rumen, Thysaniezia ovilla and intestinal Nematodes as Bunostomum phlebotomum and Bosicola radiatum.

The medicaments are administered $50 \mathrm{mg} / \mathrm{kg}$ for H1lomid and $30-35 \mathrm{mg} / \mathrm{kg}$ for Tribromine derived. The doses are not repeated. 

suffisant.

The margin for safety $(C / T=3$ à 5$)$ seems, on the whole, to be

The treatment of recent fascioliasis (six weeks or less) is difficult. With tribromine derived, the doses might be increased, so that the safety index falls. In this case, precautions are indispensable (weighing of the animals and dispensing of strictly correct doses).

\section{RESUMEN}

Estudio, en el cebú de las zonas tropicales, del poder antibelmíntico de dos compuestos dibenzenicos, derivados bromiferos de la Salicylanilida

El autor estudia el poder antihelmíntico de dos derivados bromiferos de la Salicylanilida: el Hilomid y su componente tribromifero Los dos medicamentos tienen un espectro de actividad extendido que tiene una acción sobre las Fascrola gıgantıca maduras de más de 77 semanas de edad, los Paramphistoma y los Gastrothylacidae adultos de la panza, Thysaniezia ovilla y los Nemátodos como Bunostomum phlebotomum y Bosicola radiatum.

Se administran, sin dieta previa, el Hilomid en dosis de $50 \mathrm{mg} / \mathrm{kg}$ y el derivado tribromifero en las de $30-35 \mathrm{mg} / \mathrm{kg}$. No se repeten las dosis.

El margen de seguridad varia según la edad y el estado de los animales: Parece, en el conjunto, suficiente $(\mathrm{C} / \mathrm{T}=3$ hasta 5).

Cuando se trata de distomatosis recientes, el tratamiento es más dificil. Con el Derivado tribromifero, se necesitan dosis superıores, lo que reduce otro tanto el coeficiente quimioterapeútico. En dicho caso, precauciones son necesarias (peso de los animales y administración de una dosis rigorosamente exacta).

\section{BIBLIOGRAPHIE}

« A new remedy against fluke », Vet. Rec. Suppl., 1966, 79 (1) VIII.

ARUNDEL (J. H.), "Recent advances in anthelmintics », Aust. Vet. J., 1967, 43, 455-9.

BIRGI (E.), GRABER (M.), « Mollusques pulmonés d'eau douce basommatophores, vecteurs au Tchad d'affections parasitaires du bétail. Leur élevage au laboratoire », Rev. Elev. Méd. vét. Pays trop., 1969,22 (3) : 393-408.

BORAY (J.C.), "Standardization of techniques for pathological and anthelmintic studies with Fascio la spp. », Proc. 1th. Int. Conf. world. Ass. Adv. Vet. Parasit., Hanovre (1963), 1964, pp. 34-45.

BORAY (J. C.), HAPPICH (F.A.), ANDREWS (J.C.), \& Tests on the anthelmintic efficiency of Hilomid against immature and mature Fasciola hepatica sheep », Vet. Rec., 1965, 77 (6): 175-6.

BORAY (J. C.), HAPPICH (F.A.), \& Tests on the anthelmintic efficiency of Hilomid against immature and mature $F$. hepasica in sheep and its toxicity », Vet. Rec., 1966, 79 (13) : 358-62.

BORAY (J.C.), HAPPICH (F. A.), ANDREWS (J. C.), \& Comparative chemotherapeutical tests in sheep infected with immature and mature $F$. hepatica », Vet. Rec., 1967, 80 (6) : 218-24.

BORAY (J.C.), HAPPICH (F. A.), «Standardized chemoterapeutical tests for immature and mature Fasciola hepatica infections in sheep », Aust. Vet J., 1968, 44 (2) : 72-8.

CHIROL (C. L.), DAVID (A.), «Expérimentation d'un nouveau douvicide chez les bovins», Bull. Soc. Vét. Pratique, 1967, 51 (4) : 165-73.

DANNONAY (B.), «Un traitement spécifique de la fasciolose hépatobiliaire: action fasciolıcide de dérivés de la Salicylanilide $*$, Thèse vétérinaire, Lyon, 1968, n ${ }^{0}$ 32, $71 \mathrm{p}$.

EUZEBY (J.), «Données modernes concernant le traitement et la prophylaxie des helminthoses digestives des bovins ", Rev. Méd. Vét., 1968, 119, (5) : 475-516.

GRABER (M.), FERNAGUT (R.), et OUMATIE (O.), « Helminthes des zébus adultes de la région de Maroua (Nord Cameroun) », Rev. Elev. Méd. vét. Pays trop., 1966, 19 (2) : 149-162.

GRABER (M.), «Quelques renseignements concernant le parasitısme gastro-intestinal et hépatique des animaux domestiques dans les préfectures du Sud de la République du Tchad - Possibilités de lutte ", Conf. Serv. Elev. Fort-Lamy 1969 (mars), $66 \mathrm{p}$.

GRABER (M.), BOUCHET (A.), FINELLE (P.), DESROTOUR (J.), GRENGDABO (A.), «Le parasitisme du zébu dans l'Ouest centrafricain. 2. Parasitisme des bouvillons et des adultes ", Rev. Elev. Méd. vét. Pays trop., 1969, 22 (4): 509-19.

HILDEBRANDT (J.), «Dibromo Salicylanilide Tribromo Salicylanilide. Effect against immature and mature stages of $F$. gigantica in artificially infected sheep ", Vet. Rec., 1968, 82 (24): 699-700.

I.E.M.V.T., Rap. Ann. Laboratoire Farcha, 1967, t. III, 33-37.

LIENERT (E.), «Diaphène wirkt beim Fasciola hepatica befall des rindes», Tierarzll. Umsch. 1963, 18 (2): 85-8.

NAKAMURA (R.) et collab., «Treatment of bovine fascioliasis with a salicylanilide derivate " (en (japonais) ", J. Jap. Vet. Med Ass., 1966, 19, $54-8$ et 77.

ONO (Y.), KIMURA (S.), ASAO (T.), KATAMURA (M), "Studies on the treatment of fascioliasis. VI. Anthelmintic effect of a Bromide preparation on liver. Flukes in cattle ", Jap. J. Parasit,, 1964, 13 (5) : 403-7. 